\title{
Effect of Lumbar Drainage on Incidence of Shunt-De- pendent Hydrocephalus in Patients with Subarachnoid Hemorrhage Due to Ruptured Aneurysm Who Under- went Coil Embolization
}

\author{
Soung Bin Yim¹, Yeongu Chung ${ }^{1}$, Pil Wook Chung ${ }^{2}$, Yu Sam Won ${ }^{1}$ \\ Departments of ${ }^{1}$ Neurosurgery, ${ }^{2}$ Neurology, Kangbuk Samsung Hospital, Sungkyunkwan University School of Medicine, \\ Seoul, Republic of Korea \\ Corresponding author: \\ Yu Sam Won \\ Department of Neurosurgery, \\ Kangbuk Samsung Hospital, \\ Sungkyunkwan University School \\ of Medicine, 29 Saemunan-ro, \\ Jongno-gu, Seoul 03181, \\ Republic of Korea \\ Tel: $+82-2-2001-2450$ \\ Fax: +82-2-2001-2157 \\ E-mail: yusam.won@samsung.com \\ Objective: Chronic or shunt-dependent hydrocephalus (SDH) is a hydrocephalus that develops \\ at two weeks or more after onset of subarachnoid hemorrhage (SAH). A recent study has re- \\ ported that lumbar drainage (LD) can reduce the incidence of SDH after coil embolization of \\ aneurysmal $\mathrm{SAH}$, different from previous studies. The purpose of this study was to determine \\ the effect of LD on incidence of SDH after SAH in patients who underwent coil embolization. \\ Methods: A total of 113 patients who were treated with coil embolization only for SAH from \\ January 2006 to August 2013 were investigated. They were randomly allocated into two groups \\ depending on the management protocol that included LD insertion or not. SDH was defined \\ based on Evans index and bicaudate index on computed tomography. We compared incidence \\ of SDH according to the presence and absence of LD. Results: LD was performed in 53 (46.9\%) \\ patients. Of these patients, 14 had SDH measured by Evans index $(p=0.089)$ while seven had \\ $\mathrm{SDH}$ measured by bicaudate index $(p=0.007)$. Ventriculoperitoneal shunt was performed in five \\ patients of LD group $(p=0.258)$. Conclusion: LD insertion in patients who underwent coil emboli- \\ zation reduced the risk of SDH after SAH.
}

Received: August 31, 2018

Revised: September 7, 2018

Accepted: September 10, 2018
Key Words: Endovascular procedures; Hydrocephalus; Subarachnoid hemorrhage; Ventriculoperitoneal shunt

\section{INTRODUCTION}

Hydrocephalus is one of complications after subarachnoid hemorrhage (SAH). Although previous studies have reported that its incidence has a wide range $(6 \%-67 \%)^{28)}$, recent studies have shown an incidence of $20 \%$ to $30 \%{ }^{2}$. Hydrocephalus that develops at two weeks or more after the onset of SAH is called chronic or shunt-dependent hydrocephalus (SDH) which requires surgical treatment such as ventriculoperitoneal shunt (VPS) surgery. Although the cause of hydrocephalus remains unclear, it is associated with obstruction of cerebrospinal fluid (CSF) flow with blood clots, proteins, or fibrosis blocking CSF absorption $^{2,10)}$.

In a meta-analysis of 25 studies, risk factors of SDH after SAH include increased age ( $\geq 50)$, female gender, high HuntHess grades, low Glasgow Coma Scale scores $(\leq 8)$, high Fisher grades $(\geq 3)$, acute hydrocephalus, external ventricular drainage (EVD) insertion, intraventricular hemorrhage (IVH), posterior circulation aneurysm, anterior communicating artery aneurysm, clinical vasospasm, meningitis, and rebleeding ${ }^{26)}$. Treatments for SDH after SAH include medications (such as acetazolamide and mannitol) and surgical treatments (such as lamina terminalis fenestration, VPS, and lumbar-peritoneal shunting $)^{2}$. Medications are used prior to surgical treatment rather than reducing incidence of SDH. Although surgical treatments are associated with high incidence of complications, they are preferred treatments for SDH, especially VPS which is widely used ${ }^{29)}$.

Current treatment options for SAH due to ruptured aneurysm are surgical clipping and coil embolization. According to the Korean Clinical Practice Guidelines for aneurysmal SAH published in 2018, coil embolization is beneficial for short-term treatment outcomes, but detrimental for long-term ones. Therefore, coil embolization is not the first treatment option for every $\mathrm{SAH}$, but selected as a primary treatment if both surgical clipping and coil embolization are available ${ }^{4}$. However, many neurosurgeons believe that surgical treatments such as hematoma evacuation, widening the opening of cisterns, and fenestration of the lamina terminalis performed with surgical clipping can improve CSF flow and reduce the incidence of SDH after 
SAH. In 2009, Komotar et al. ${ }^{15)}$ suggested that there was no significant difference in the prevalence of SDH between patients who had undergone fenestration of the lamina terminalis and those had not. Several studies have reported differences in the incidence of SDH after SAH between clipping surgery and coil embolization ${ }^{5,6,11,16,27)}$. In 2010, Yong et al. ${ }^{28)}$ reported that lumbar drainage (LD) for patients with good grades (Hunt and Hess grades I-III) could reduce the incidence of SDH after coil embolization of aneurysmal SAH (3.1\%). Such incidence was lower than those reported in previous studies (19.0 and $19.6 \%)^{28)}$. However, the study of Yong et $\mathrm{al}^{28)}$ was performed in patients with LD only. Thus, further research is needed to compare outcomes between patients with and without LD.

Whether VPS operation should be performed in patients with hydrocephalus confirmed by images such as computed tomography (CT) is often determined by surgeon's subjective judgment. It is inaccurate to objectively evaluate the incidence of SDH as the number of patients with VPS surgery. Thus, the purpose of this study was to determine the effect of LD on the incidence of SDH confirmed by imaging in patients with SAH due to ruptured cerebral aneurysm who underwent coil embolization.

\section{MATERIALS AND METHODS}

\section{Patients}

A total of 113 patients treated with coil embolization only for SAH due to non-traumatic ruptured aneurysm who visited emergency room from January 2006 to August 2013 were reviewed, including 52 males and 61 females. Their mean age was 56.5 years (range, 30-86 years). Of these patients, $46 \mathrm{had}$ hypertension, eight had diabetes mellitus, and 11 were smokers. Information about Hunt and Hess grade, Fischer grade, and aneurysm location for these patient is shown in Table 1.

Initial non-contrast CT was performed for all patients for diagnosis of SAH. Aneurysm was confirmed by CT angiography or conventional cerebral angiography. All patients were admitted through the emergency room and coil embolization was performed within $48 \mathrm{hr}$ after admission by two neuro-intervention radiologists. After intervention, standard post SAH management was performed in intensive care unit, including prevention of rebleeding and treatment of vasospasm and hydrocephalus. Patients were randomly assigned to post SAH management protocol included LD insertion or not. LD insertion was performed within $24 \mathrm{hr}$ after coil embolization. Amount of CSF drainage was 150 to $200 \mathrm{cc} /$ day. Drainage catheter was maintained for a week.

In all patients, follow up CT was performed at one week, two weeks, and one month. SDH was defined based on measurement of Evans index and bicaudate index at the axial cutting of CT at two weeks or one month. All shunt operations were performed with a VPS. Hydrocephalus on initial CT and mental state change was defined as acute hydrocephalus. If necessary, EVD insertion was performed by burr hole craniotomy at right or left or both Kocher's points before coil embolization in emergency room, intensive care unit, or operating room. Transcranial Doppler was also used at intervals of two to three days for 2 weeks after coil embolization to observe vasospasm.

\section{Statistical Analysis}

Statistical analysis was performed using SPSS version 25.0 for Windows(SPSS Inc., Chicago, IL, USA). Pearson's $X^{2}$ test

Table 1. Characteristics of patients $(n=113)$ with coil embolization

\begin{tabular}{|c|c|}
\hline & Number of patients, $\mathrm{n}(\%)$ \\
\hline Mean age, year & $56.5(30-86)^{*}$ \\
\hline \multicolumn{2}{|l|}{ Sex } \\
\hline Male & $52(46.0)$ \\
\hline Female & $61(54.0)$ \\
\hline Hypertension & $46(40.7)$ \\
\hline DM & $8(7.1)$ \\
\hline Smoking & $11(9.7)$ \\
\hline \multicolumn{2}{|l|}{ Hunt and Hess grade } \\
\hline I & $3(2.7)$ \\
\hline$\|$ & $52(46.0)$ \\
\hline III & 32 (28.3) \\
\hline IV & $22(19.5)$ \\
\hline V & $4(3.5)$ \\
\hline \multicolumn{2}{|l|}{ Fischer grade } \\
\hline I & $0(0.0)$ \\
\hline$\|$ & $39(34.5)$ \\
\hline III & $38(33.6)$ \\
\hline IV & $36(31.9)$ \\
\hline \multicolumn{2}{|l|}{ Aneurysm location } \\
\hline \multicolumn{2}{|l|}{ ICA } \\
\hline PCOM & $16(14.2)$ \\
\hline Others & $14(12.4)$ \\
\hline \multicolumn{2}{|l|}{ ACA } \\
\hline $\mathrm{ACOM}$ & $41(36.3)$ \\
\hline Others & $7(6.2)$ \\
\hline $\mathrm{MCA}$ & $16(14.2)$ \\
\hline BA, VA, SCA, PICA & $19(16.8)$ \\
\hline
\end{tabular}

The data is presented as number (\%).

DM: diabetes mellitus; ICA: internal carotid artery; PCOM: posterior communicating artery; $A C A$ : anterior cerebral artery; $A C O M$ : anterior communicating artery; MCA: middle cerebral artery; $\mathrm{BA}$ : basilar artery; VA: vertebral artery; SCA: superior cerebellar artery; PICA: posterior inferior cerebellar artery.

"The range of patient ages. 
Table 2. Differences according to the presence of lumbar drainage insertion

\begin{tabular}{lccc}
\hline \hline & Without LD groups $(\mathrm{n}=60), \mathrm{n}(\%)$ & With LD groups $(\mathrm{n}=53), \mathrm{n}(\%)$ & $\mathrm{p}$-value \\
\hline $\mathrm{SDH}$ & & & \\
Evans' index & $25(41.7)$ & $14(26.4)$ & 0.089 \\
Bicaudate index & $21(35.0)$ & $7(13.2)$ & 0.007 \\
Shunt operation & $10(16.7)$ & $5(9.4)$ & 0.258 \\
Acute hydrocephalus & $21(35.0)$ & $18(34.0)$ & 0.908 \\
IVH & $21(35.0)$ & $15(28.3)$ & 0.446 \\
EVD insertion & $17(29.3)$ & $14(26.4)$ & 0.820 \\
Vasospasm & $18(30.0)$ & $12(22.6)$ & 0.307 \\
\hline
\end{tabular}

The data is presented as number (\%).

LD: lumbar drainage; SDH: shunt-dependent hydrocephalus; IVH: intraventricular hemorrhage; EVD: external ventricular drainage.

was used to compare incidence of SDH between groups with or without LD insertion.

\section{RESULTS}

Of 113 patients, 60 patients had no LD insertion while 53 patients had LD insertion (Table 2). Based on SDH measured by Evans' index, there were 25 patients in the group without $\mathrm{LD}$ and 14 patients in the group with $\mathrm{LD}(\mathrm{p}=0.089)$. There were statistically significant differences in SDH measured by bicaudate index: 21 patients in the group without LD versus 7 patients in the group with $\mathrm{LD}(\mathrm{p}=0.007)$. Shunt operation was performed for ten patients in the group without LD and five patients in the group with $\mathrm{LD}$, showing no significant difference between the two groups $(\mathrm{p}=0.258)$. Acute hydrocephalus, IVH, EVD insertion, or vasospasm was not significantly different between the two groups (Table 2).

\section{DISCUSSION}

\section{SDH}

Reported incidence of hydrocephalus as one of complications of SAH ranges from $6 \%$ to $67 \%{ }^{21)}$. de Oliveira et al. have reported that patients treated with surgical clipping have significantly lower risk of SDH than patients treated with coil embolization. However, other studies suggest that there is no significant difference of risk of SDH between clipping and coiling ${ }^{6,11)}$. According to a recent large scale meta-analysis, SDH was detected in $17.4 \%$ of patients with SAH after coil embolization and clipping surgery ${ }^{16)}$. There was no significant difference in incidence of SDH between the two technique groups ${ }^{16)}$. In 2015, Yamada et al. ${ }^{27)}$ announced that SDH in patients who underwent surgical clipping after $\mathrm{SAH}$ was twice as high as that in those who underwent coil embolization. Therefore, this issue remains controversial. Hydrocephalus after SAH was first suggested by Bagley ${ }^{1)}$ in 1928 and first defined by Vale et $\mathrm{al}^{21)}$ in 1997. Depending on the time of onset, hydrocephalus after SAH could be divided into acute (0-3 days post SAH), subacute (4-13 days post SAH) and chronic hydrocephalus ${ }^{21)}$. Chronic hydrocephalus occurs after two weeks of SAH. It is also referred to as SDH. Unlike acute hydrocephalus that can be diagnosed based on symptoms such as headache, vomiting, and change of consciousness, SDH is difficult to be diagnosed based on symptoms ${ }^{2}$. To diagnose SDH, image such as CT is needed to confirm that the ventricle size is enlarged anatomically").

Several methods have been devised to measure ventricle size to diagnose hydrocephalus on CT. The most widely used marker is the bicaudate index. In 1970, Galera and Greitz ${ }^{8)}$ compared the maximum width of frontal horns of lateral ventricle to the same axial level. In 1979, Vassilouthis and Richardson ${ }^{23)}$ suggested radiological marker of ventricle size measuring the width of lateral ventricles at the level of the foramen of Monro as a fraction of the transverse inner diameter of the skull at the same level. They defined hydrocephalus if this ratio (the width of lateral ventricles: the inner diameter of the skull) was more than 1:4. In 1985, van Gijn and colleagues ${ }^{22)}$ proposed the bicaudate index as a linear measurement of ventricular size: the width of frontal horns at the level of the caudate nuclei divided by the diameter of the brain at the same level (Fig. 1A). They also suggested that diagnosis of hydrocephalus should be made when bicaudate index was more than ageadjusted $95^{\text {th }}$ percentile ${ }^{22)}$.

Evans Index is a ratio of the transverse diameter of the anterior horn of lateral ventricles to the largest internal diameter of the skull first described by Evans ${ }^{7}$ in 1942. Initially, it was a method to measure ventricular size using pneumocephalus grams in pediatric patients. Later, it was adjusted for CT scans. This index is usually used in normal pressure hydrocephalus $(\mathrm{NPH})$. Hydrocephalus can be diagnosed if this index is greater than 0.3 in international NPH guidelines (Fig. 1B) ${ }^{20)}$. In our study, we defined hydrocephalus using bicaudate index and Evans' index measured with CT for patient groups. 


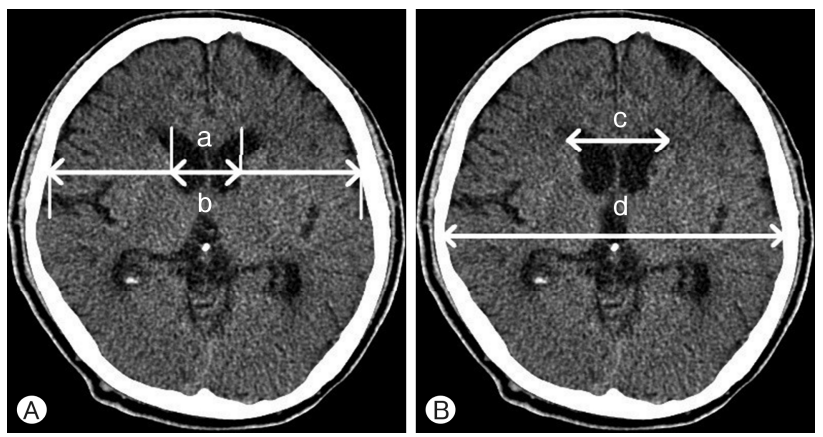

Fig. 1. Axial view of brain non-contrast computed tomography demonstrating how to measure shunt dependent hydrocephalus. (A) Bicaudate index. " $a$ " is the distance between caudate nuclei and " $b$ " is the width of the brain at the same level. The ratio of $a / b$ is bicaudate index, (B) Evans' index. "C" is the transverse diameter of the anterior horns of the lateral ventricles and " $\mathrm{d}$ " is the greatest internal diameter of the skull. The ratio of $c / d$ is Evans index.

\section{Etiology of Hydrocephalus after SAH}

Several hypotheses for development of SDH after SAH have been proposed and the most promising cause is change in CSF flow dynamic ${ }^{28)}$. Approximately $30 \%$ of SAH patients are known to have permanent impairment of CSF diversion ${ }^{16)}$. Despite extensive studies about CSF dynamic changes in acute and chronic hydrocephalus, the exact mechanism by which hydrocephalus occurs after SAH remains poorly understood ${ }^{10)}$. The mechanism of recent trends seems to be related to the pathogenesis of acute and chronic hydrocephalus, damage associated with arachnoid granulations, and brain tissue ${ }^{2)}$. In this mechanism, inflammatory reaction and the resulting fibrosis process can disturb CSF flow outward from arachnoid granulations to sinus. After SAH, the subarachnoid space is filled with blood cells and products. Subsequently, proliferation of leptomeninx is observed with hemosiderin deposits. In arachnoid granulations, one major pathological mechanism is that blood clots and corresponding products can block the outflow tract of CSF (obstruction as non-communicating type). Another one is fibrosis of arachnoid membrane (decreased absorption as communicating type) $)^{2,10)}$.

Acute hydrocephalus is attributed to blood clots within ventricles and aqueduct that can prevent CSF flow out of the cranial vault. Inflammation also induces acute hydrocephalus by disrupting the blood-brain barrier ${ }^{3)}$. Recent studies have suggested that acute and chronic hydrocephalus have similar pathophysiology and that obstruction is not the only cause of acute hydrocephalus ${ }^{19)}$. Other studies have assumed that blood clots can trigger hypersecretion of CSF and fibrosis of arachnoid granulations, leading to long-term communicating hydrocephalus rather than simply aqueduct obstruction ${ }^{12)}$. More research is needed to discover and explain the etiology of acute hydrocephalus after SAH. In chronic hydrocephalus, it is considered a communicating type attributed to fibrosis and adhesions of leptomeningeal and arachnoid granulations becaus e intracranial pressure is not increased in patients, with sufficient evidence emerging in the pathway of fibrosis ${ }^{2,9)}$.

\section{Principle of LD}

Considering the pathophysiology of hydrocephalus after $\mathrm{SAH}$, it is possible to reduce the incidence of hydrocephalus by irrigation or drainage of blood clots and fibrosis-inducing substance. In open surgery, it can be done by irrigating the basal cistern or the opening of the lamina terminalis. However, this cannot be performed in patients with coil embolization. Several studies have reported that this treatment does not improve neurological outcome or decrease the rate of hydrocephalus ${ }^{15)}$. Drainage of CSF using EVD is usually used for acute hydrocephalus. However, it appears to promote stasis of blood clots in the basal cistern instead of clearance. It has been reported that drainage of CSF via ventricle can produce a high rate of $\mathrm{SDH}^{13,24)}$. Because blood is heavier than $\mathrm{CSF}$, it will remain in the basal cistern. This has been observed in patients treated with both EVD and $\mathrm{LD}^{14)}$. CSF drainage through EVD is effective in the ventricle. However, blood can remain in lumbar compartment ${ }^{17)}$. LD removes subarachnoid blood more rapidly than EVD and reduces the need for VPS ${ }^{18)}$. Thus, removing blood with a LD can be suggested as an appropriate treatment option after $\mathrm{SAH}^{25)}$.

Based on this background, we compared the incidence of SDH with or without LD in patients who underwent coil embolization for $\mathrm{SAH}$. As a result, the incidence of $\mathrm{SDH}$ measured by bicaudate index was $13.2 \%$ in patients with $\mathrm{LD}$ and $35.0 \%$ in patient without LD (Table 2), showing significant $(\mathrm{p}=0.007$ ) different between the two groups. Our results confirmed that LD could reduce the incidence of SDH after SAH. Thus, LD is an effective treatment for $\mathrm{SAH}$.

\section{Limitation}

This study has some limitations. First, the number of patients enrolled for this study was small. In addition, patients with poor neurological state (Hunt and Hess grade IV and V) tended to have EVD insertion additionally because of IVH or acute hydrocephalus. Also, criteria for diagnosis of hydrocephalus by images or symptoms and the indication of VPS operation varied depending on each neurosurgeon.

\section{CONCLUSION}

Difference in incidence of SDH after SAH between clipping surgery and coil embolization remains controversial. According to recent guidelines for aneurysmal $\mathrm{SAH}$, coil embolization is preferred. In our study, LD insertion in these patients reduced the risk of SDH after SAH. However, further studies enrolling 
a large number of patients are needed to confirm results of this study.

\section{CONFLICT OF INTEREST}

No potential conflict of interest relevant to this article was reported.

\section{REFERENCES}

1. Bagley C, Jr: Blood in the cerebrospinal fluid resultant functional and organic alterations in the central nervous system a. experimental data. Arch Surg 17:18-38, 1928

2. Chen S, Luo J, Reis C, Manaenko A, Zhang J: Hydrocephalus after Subarachnoid Hemorrhage: Pathophysiology, Diagnosis, and Treatment. Biomed Res Int 2017:8584753, 2017

3. Chen S, Yang Q, Chen G, Zhang JH: An update on inflammation in the acute phase of intracerebral hemorrhage. Transl Stroke Res 6:4-8, 2015

4. Cho WS, Kim JE, Park SQ, Ko JK, Kim DW, Park JC, et al.: Korean clinical practice guidelines for aneurysmal subarachnoid hemorrhage. J Korean Neurosurg Soc 61:127-166, 2018

5. de Oliveira JG, Beck J, Setzer M, Gerlach R, Vatter H, Seifert $\mathrm{V}$, et al.: Risk of shunt-dependent hydrocephalus after occlusion of ruptured intracranial aneurysms by surgical clipping or endovascular coiling: a single-institution series and meta-analysis. Neurosurgery 61:924-933, 2007

6. Dehdashti AR, Rilliet B, Rufenacht DA, de Tribolet N: Shuntdependent hydrocephalus after rupture of intracranial aneurysms: a prospective study of the influence of treatment modality. J Neurosurg 101:402-407, 2004

7. Evans WA, Jr.: An encephalographic ratio for estimating ventricular enlargement and cerebral atrophy. Arch Neurol Psychiatry 47:931-937, 1942

8. Galera R, Greitz T: Hydrocephalus in the adult secondary to the rupture of intracranial arterial aneurysms. J Neurosurg 32: 634-641, 1970

9. Gao C, Du H, Hua Y, Keep RF, Strahle J, Xi G: Role of red blood cell lysis and iron in hydrocephalus after intraventricular hemorrhage. J Cereb Blood Flow Metab 34:1070-1075, 2014

10. Germanwala AV, Huang J, Tamargo RJ: Hydrocephalus after aneurysmal subarachnoid hemorrhage. Neurosurg Clin N Am 21:263-270, 2010

11. Jartti P, Karttunen A, Isokangas JM, Jartti A, Koskelainen T, Tervonen O: Chronic hydrocephalus after neurosurgical and endovascular treatment of ruptured intracranial aneurysms. Acta Radiol 49:680-686, 2008

12. Kanat A, Turkmenoglu O, Aydin MD, Yolas C, Aydin N, Gursan $\mathrm{N}$, et al.: Toward changing of the pathophysiologic basis of acute hydrocephalus after subarachnoid hemorrhage: a preliminary experimental study. World Neurosurg 80:390-395, 2013

13. Kasuya H, Shimizu T, Kagawa M: The effect of continuous drainage of cerebrospinal fluid in patients with subarachnoid hemorrhage: a retrospective analysis of 108 patients. Neurosurgery 28:56-59, 1991

14. Klimo P, Jr., Kestle JR, MacDonald JD, Schmidt RH: Marked reduction of cerebral vasospasm with lumbar drainage of cere- bro spinal fluid after subarachnoid hemorrhage. J Neurosurg 100:215-224, 2004

15. Komotar RJ, Hahn DK, Kim GH, Starke RM, Garrett MC, Merkow MB, et al.: Efficacy of lamina terminalis fenestration in reducing shunt-dependent hydrocephalus following aneurysmal subarachnoid hemorrhage: a systematic review. Clinical article. J Neurosurg 111:147-154, 2009

16. Li H, Pan R, Wang H, Rong X, Yin Z, Milgrom DP, et al.: Clipping versus coiling for ruptured intracranial aneurysms: a systematic review and meta-analysis. Stroke 44:29-37, 2013

17. Maeda Y, Shirao S, Yoneda H, Ishihara H, Shinoyama M, Oka F, et al.: Comparison of lumbar drainage and external ventricular drainage for clearance of subarachnoid clots after Guglielmi detachable coil embolization for aneurysmal subarachnoid hemorrhage. Clin Neurol Neurosurg 115:965-970, 2013

18. Ormond DR, Dressler A, Kim S, Ronecker J, Murali R: Lumbar drains may reduce the need for permanent CSF diversion in spontaneous subarachnoid haemorrhage. Br J Neurosurg 27:171-174, 2013

19. Saliou G, Paradot G, Gondry C, Bouzerar R, Lehmann P, Meyers $\mathrm{ME}$, et al.: A phase-contrast MRI study of acute and chronic hydrodynamic alterations after hydrocephalus induced by subarachnoid hemorrhage. J Neuroimaging 22:343-350, 2012

20. Toma AK, Holl E, Kitchen ND, Watkins LD: Evans' index revisited: the need for an alternative in normal pressure hydrocephalus. Neurosurgery 68:939-944, 2011

21. Vale FL, Bradley EL, Fisher WS, 3rd: The relationship of subarachnoid hemorrhage and the need for postoperative shunting. J Neurosurg 86:462-466, 1997

22. van Gijn J, Hijdra A, Wijdicks EF, Vermeulen M, van Crevel $\mathrm{H}$ : Acute hydrocephalus after aneurysmal subarachnoid hemorrhage. J Neurosurg 63:355-362, 1985

23. Vassilouthis J, Richardson AE: Ventricular dilatation and communicating hydrocephalus following spontaneous subarachnoid hemorrhage. J Neurosurg 51:341-351, 1979

24. Widenka DC, Wolf S, Schürer L, Plev DV, Lumenta CB: Factors leading to hydrocephalus after aneurysmal subarachnoid hemorrhage. Neurol Neurochir Pol 34:56-60, 2000

25. Wolf S: Rationale for lumbar drains in aneurysmal subarachnoid hemorrhage. Curr Opin Crit Care 21:120-126, 2015

26. Xie Z, Hu X, Zan X, Lin S, Li H, You C: Predictors of shuntdependent hydrocephalus after aneurysmal subarachnoid hemorrhage? A systematic review and meta-analysis. World Neurosurg 106:844-860.e846, 2017

27. Yamada S, Ishikawa M, Yamamoto K, Ino T, Kimura T, Kobayashi S: Aneurysm location and clipping versus coiling for development of secondary normal-pressure hydrocephalus after aneurysmal subarachnoid hemorrhage: Japanese Stroke DataBank. J Neurosurg 123:1555-1561, 2015

28. Yong CI, Hwang SK, Kim SH: The role of lumbar drainage to prevent shunt-dependent hydrocephalus after coil embolization for aneurysmal subarachnoid hemorrhage in good-grade patients. J Korean Neurosurg Soc 48:480-484, 2010

29. Zaidi HA, Montoure A, Elhadi A, Nakaji P, McDougall CG, Albuquerque FC, et al.: Long-term functional outcomes and predictors of shunt-dependent hydrocephalus after treatment of ruptured intracranial aneurysms in the BRAT trial: revisiting the clip vs coil debate. Neurosurgery 76:608-613, 2015 